\title{
Design of Fuzzy-PID controller using logarithmic approximations for IMC-LFC Model
}

\author{
Arunsai $\mathrm{V}^{1}$, Srividya Devi $\mathrm{P}^{2 *}$, and Vijaya Laxmi. $\mathrm{U}^{3}$ \\ ${ }^{1}$ Research Scholar, EEE Dept., GRIET, Hyderabad, Telangana \\ ${ }^{2}$ Associate Professor, EEE Dept., GRIET, Hyderabad, Telangana \\ ${ }^{3}$ Assistant Professor, EEEDept., GRIET, Hyderabad, Telangana
}

\begin{abstract}
This paper provides a Artificial Intelligent controller layered to analyze the power system's logarithmic load frequency control problems. The control system ensures the preservation of steady frequency errors and of inadvertent bonding in a tolerance limit. The proposed control system was developed for a two-area hydrothermal heating system. The approach to control design is based on the two-degree internal model control (2DOF), which combines the fuzzy control technique for model order reduction to increase frequency excursions. Different techniques of the two-area reheating hydraulic energy system are integrated into the scheme and a correct combining of energy resources is seen in different operational conditions as a pragmatic solution. The IMC- FUZZY-PID architecture of the reduced order model, proposed with the original high order framework, delivers strong dynamic response and load sturdiness.
\end{abstract}

\section{Introduction}

Due to quick load disturbances, uncertainty of technical constants, operational differences, etc, performance can deteriorate in a broad power system. A ruggedness and reliability of a load frequency controller (LFC) for hugescale systems are therefore extremely critical. However, due to its complexity, non-linearity and higher order, it is very tough to build the powerful controller in a wideranging, power system. a fundamental burden of a systems in a current power system scenario is maintaining equilibrium between electricity generation and a demand for electricity, in order to provide consumers with high-quality and reliable energy. Differences in energy demand can influence a power flow of a connecting line connecting control areas as well as a frequency of electrical services. As the result, a Large Frequency Controller (LFC) is configured to organize a system frequency, to change a generator units based principally on area control error, to zero under an infinite modulation of active electricity generation and therefore to balance a whole system with a load power well. This is because the better composition controller would have better control.

This can therefore be seen as a purpose enhancement or robust control problem [1-8] that counteracts unexpected disturbances, uncertain parameters, and can also operate effectively on a tie line under the defined overhead, set-up time, frequency and power variation [1]. Antipathy [8] proposed in 2009 to use multi-target evolutionary algorithms, with a Dead Band Governor and superconductive magnetic energy stores units (MESS), the decentralized LFC of interconnected energy

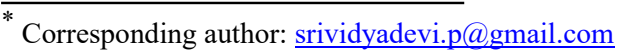

systems(MOEA). For disrupted multifaceted power systems, the localized PID-LFC, where a power system's LFC performance depends on a locus on a stability border, was proposed by Sahaj et al. [9]. A design strategy, which was seen in the LFC literature to this day [9], causes parametric uncertainty. Uncertainty parameters in Swatietal were evaluated. [11] Considering the fractional order control principle ; a solid controller was designed to be similar to an internal model - proportional integrated derivatives [11] (IMCPID).

In addition the method [12] for the application of load theory following an automatic power generation control has been seen in a reconstructed power system scenario. In light of contractual violations, a proposed hydrothermal energy system [12] is validated. The test device for various loads is an additional three-area unified electricity system [13]. There are several problems with goal optimization by improving the algorithm with a cluster-based set [14] with the proposal for the stabilization of the two-decker Multi-Driving Systems (SSSC), the magnetic energy storage (SMES), the thyristor control transfer, and more. Different technological optimizations have been suggested over the past decades [16-20] to maximize control gains in multi-area power systems to produce three degrees of 3DOF-PID and cascade controls [19,20]. A victorious 3DOF PID controller $[16,18]$ and a 3DOF ID controller [17] were respectively developed for different four/twoscale thermal and two-scale hydrothermal systems in the application of a highly optimized bio-geographic technique.

In addition, the data-based method [21] for repetition parameter adherence in the 3-DOF control structure was developed to improve a tracking efficiency. There are 
three aspects to a database tuning process. First, use of input and output information where a cost is eliminated while the precise model is obtained. It can also be used as the controller tuning tool. Third, it provides better monitoring results without any structural changes. The Jack Feed forward (JFF) controller [20], based on pliable and rigid body mode, is also proposed for flexible motion systems. The three-phase device study has been conducted with reduced order modeling [23] to correct electric power factor, With three internal, The control technology uses reduced control current controllers and the external voltage loop. It offers fast temporary response, easy control and enhanced strength. There may be difficulties to design the load frequency control in the analysis, synthesis and identification of a mathematical model for a high-order power system. Typically the unattainable control system creates a higher order method which results in slow reactions with further calculations. Low order model with main qualitative characteristics such as a constant time, damping ratio, stability, etc., is therefore suggested to approximate it with the original high-order method. Therefore, several researchers have suggested different methods to reduce the range of the model's broad-scale energy system model in order to match the number of time domain [24$28]$ and frequency domain reductions in the model order [29]. According to its application, a standard of the reduction method is measured [30-31]. The invention of the LFC power system, One of the key aims to minimize order is to efficiently manage an original high control structure, to ensure that an overall system is in order and easy to understand [32-33]. Due to the reduction of the order model, the approach is therefore more understandable. In addition, some benefits of reduced orders are 1) less work to simulate the system by the computer. 2) The configuration of the controller is more precise numerically. 3) Capable of clearer laws of power. The key aims of this paper are as follows in the sense of the above discussion : It create less computationally viable controls. (ii) the design of order models is decreased with a logarithmic approach which monitors a non-reduction part associated with an original model's output response. (iii) The creation of a two-degree controller for the most reduced order models and original internal model control models (2DOF-IMC). (iv) Apply both the original high-order and reducedorder scheme to designed controls. (v) Precision check optimized for the controller.

\section{Load frequency control design, pid structure, tuning constants}

\subsection{Load frequency control via IMC-PID design}

An internal model control system is one of the most common frequency control techniques in the field of process control. The basic structure of the IMC G (s) is a managed facility ; $\mathrm{GM}(\mathrm{s})$ are a model plant ; $\mathrm{D}(\mathrm{s})$ are load ruptures ; and $\mathrm{Q}(\mathrm{s})$ is the IMC controller configuration. In the event that a controller $Q_{a}(s)$ is an equivalent of an inverse model, i.e. $Q_{a}(s)$ $=G_{a}(s)-M_{a}(s)$ and a closed loop, in Figure 1. (s). In fact, an optimal controller cannot be achieved because of the following problems.

a) A controller $Q_{a}(s)=G_{M}^{-}(s)$ has the RHP pole. When a model has Right -Half plane (RHP) occurs zero

b) A controller $Q_{a}(s)=G_{M}^{-}(s)$ will be prophetic and may not be able to understand. When the model has time delay.

c)A controller $Q_{a}(s)=G_{M}^{-}(s)$ will not be suitable, that may require exponentially small high frequency disturbances with changing variables and they may not be physically understood. If the model is accurate.

d) Dual (open and close loop) stability does not occur and a closed-loop system will be unsteady for a controller $Q_{a}(s)=G_{M}^{-}(s)$ when $\mathrm{G}(\mathrm{S})$ is not equal to $G_{M}(s)$

Therefore, IMC design as shown in Fig 1 was done by making a GM(s) in two parts and examining one part with a desired filter in order to overcome above problems. IMCPID architecture is considered here, too, for robust and stable controllers. A following is given an IMC-PID design process $[1,2]$.

Separate the plant model GM(s) into two parts.

$G_{M}(s)=P_{M}(s) P_{A}(s)$

Where, PA(s) included all non-minimum (noninvertible) phase components in a plant model, i.e. all RHP zeros and time delays (right half plane). A PM(s) factor is the minimum and invertible step.

2) Design the set point-tracking IMC controller

The IMC controller $\mathrm{Qa}(\mathrm{s})$ is choosed as inverse of minimum phase component (invertible), i.e. . . . $Q_{a}(s)=P_{M}^{-1}(s)$. Component failure can lead to inverted problems with system instability. Also, PMs have poles on a left side of a plane, so it is evident that $Q_{a}(s)$ are incorrect and want to be properly implemented.

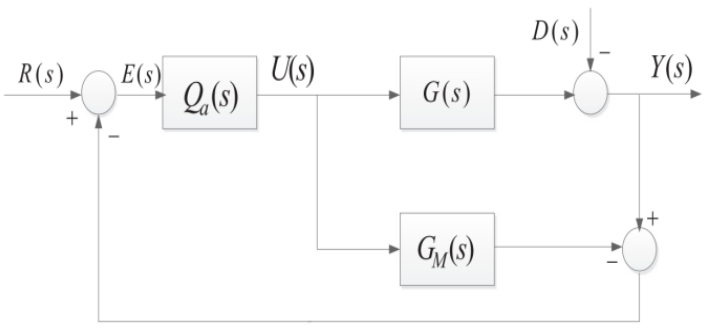

Fig.1 Internal Model Control structure for PID.

Can refuse load disturbances or, if any, model mismatch. The low pass filter feature is then used to stabilize, stabilize and correct a controller. Therefore it is written as IMC controller

$Q_{a}(s)=R_{M}^{-1}(s) \frac{1}{(1+\beta s)^{r}}$ 
Where, $\beta$ is the tuning parameter such that a desired set point response is $(1+\beta s)^{-r}$, and $\mathrm{r}$ is a relative degree of $\mathrm{P}(\mathrm{s}) \mathrm{M}(\mathrm{s})$ ?

Thus, the control operation can achieve good tracking efficiency by a use of IMC-controller Q(s). Often an output of load disruption refusal is not satisfactory [1] by $\mathrm{Q}(\mathrm{s})$ a... Therefore, the second controller is deliberated to restore a device output caused by disruptive rejection. If there is no model mistake, an ideal controller can be achieved. Nevertheless, process-model mistakes are normal. In this case, it was assumed that a GM(s) model represents a $\mathrm{G}(\mathrm{s})$ process exactly, that means that a Process Model has all an earlier model features. In realworld practise, process models may mix up with an earlier procedure ; thus, feedback controllers, i.e., $Q_{d}(s)$, are built to mitigate mismatching effects.

In a configuration of an additional $Q_{d}(s)$ regulation is as follows : Two degree of freedom-internal model control structure :

IMC controller $Q_{d}(s)$ is configured for disruptionrejecting in a form provided [1].

$Q_{d}(s)=\frac{1+\alpha_{1} s+\alpha_{2} s^{2}+\alpha_{3} s^{3}+\ldots \ldots+\alpha_{m} s^{m}}{\left(1+\lambda_{d} s\right)^{m}}$

Where, $\lambda_{d}$ is the tuning parameter and $\mathrm{m}$ is a total number of poles of a plant model GM(s).

$T_{d}$ Must be properly adjusted so that $Q_{d}(s)$ can unsteady poles caused by load disruption or model mix if any to remove disruptions or model mismatch if any. A poles to be cancelled must then be $p_{1}, p_{2} \ldots p_{m}$ and a coefficients (i.e. $\alpha_{1}, \alpha_{2} \ldots . \alpha_{m}$ ) must be cancelled.

$1-G_{M}(s) Q_{a}(s) Q_{d}(s)_{s=-p_{1}, p_{2, \ldots}, p_{m}}=0$

The slow process poles $p_{1}, p_{2} \ldots p_{m}$ which increases a controls load-rejection efficiency, are here rejected by $\alpha$, an additional degree of freedom. A discussion above can be based on a fact that a structure of 2D OFFIMC is similar to traditional two-degree free feedback structure as shown in Fig. 2

$$
Q(s)=\frac{Q_{a}(s) Q_{d}(s)}{1-G_{M}(s) Q_{a}(s) Q_{d}(s)}
$$

Further approximated IMC-PID controller, i.e., Q (s) PID [1] is given below.

$$
Q_{P I D}(s)=k_{p}+\frac{k_{i}}{s}+k_{d} s
$$

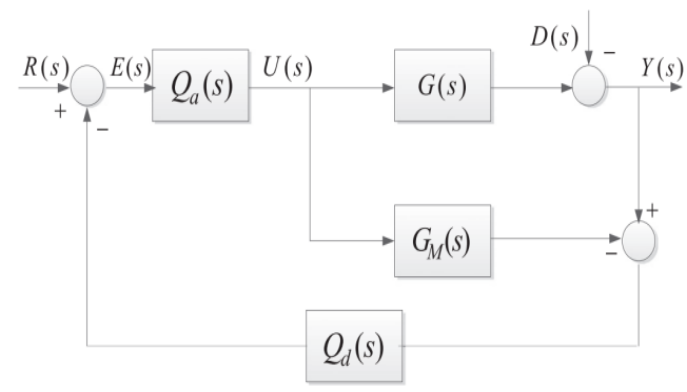

Fig. 2 Two Degree of Freedom-Internal Model Control structure

Applications of IMC structures simply need an understanding of a high-speed transmission mechanism if GM models are power systems that are high-speed and incidental to power control systems. In the next section, we will also explore a method for the reduction of the model order. The PID control scheme is stress- free and can be applied and managed in practice, so that an IMC structure can be transferred to a default PID control structure.

The basic procedure for obtaining PID parameters from an IMC controls consists in extending an ultimate controller Q(s) i.e. Eq. (5). This process of expansion is long, but results are often normal. Even after PID constants are received, often fine tuning may be required to get a perfect controller. An IMC TUNING [1] can be used for a detailed procedure. Furthermore, IMC-PID tuning is best observed and works best for steady processes only, but additional compensators may be needed for a design of unstable processes.

\subsection{Conventional PID controller structure}

The graph of a shape of a controller. A control structure of a Power System is categorized by the feedback path control unit, the controller, simulated MPG (s) dM and reduced simulated MPG(s) dRM. A loop inside a model makes use of a variation between frequency deviation and load disturbance. This discrepancy is usually referred to as the mistake, which characterizes a consequence of sudden disruption and plant/model malfunction if it happens. A most approved control technique is a proportional integral derivative (PID) controller where functional feedback loops are based on PID control or the number of small deviations. A PID control technology can be approached and viewed in various ways from as a tool that can be used with several true laws and can also be systematically progressed.

$u(t)=k_{p} v(t)+k_{i} \int v(t) d t+k_{d} \frac{d v(t)}{d t}$

$$
\begin{aligned}
\Delta f(s) & =u(s) G_{k M}(s) \\
\Delta f(s) & =u(s) G_{k R M}(s)
\end{aligned}
$$


$\Delta f(s) / \Delta p_{k M}(s)=u(s) G_{k M}(s) /\left(1+u(s) G_{k M}(s)\right)$

$\Delta f(s) / \Delta p_{k R M}(s)=u(s) G_{k R M}(s) /\left(1+u(s) G_{k R M}(s)\right)_{--}$

Here, $\Delta p_{k M}(s)$ is the load disturbance for earlier system model and $\Delta p_{d R M}(s) \mathrm{dRM}$ is a load disturbance for reduced system

$$
u(s) G_{k R M}(s)=\left(k_{p}+\frac{k_{i}}{s}+k_{d} s\right) G_{d R M}(s)
$$

\subsection{Tuning scheme for PI for constant with FUZZY Controller}

By using a Routh- table as expressed in this section. A range of controller gains $k_{p}, k_{i}$ and $k_{d}$ was calculated (11). Change each of $k_{p}, k_{i}$ and $k_{d}$ (manually) until an overall answer is desired. [5]. [5]. a use of Routh Table to define a system's stability range, however, is restricted since only one variable is determined at the single point. PI controller here

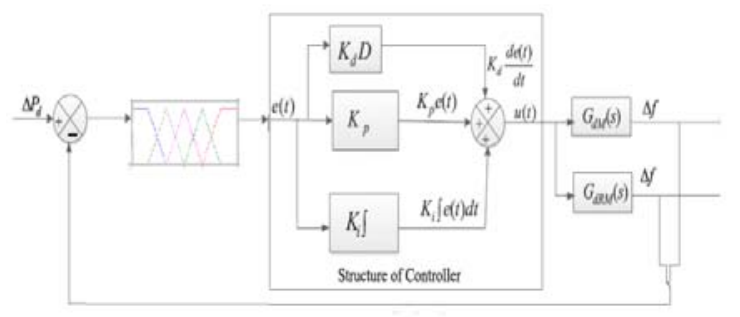

Fig. 3 Fuzzy Controller structure for IMC model

It is essential to establish their relationship first with a PID controller and then use a Routh Table to decide stability conditions. a formulation Routh recurrence is provided.

$$
\begin{aligned}
& m_{0}=\frac{x_{0}}{y_{0}}\left\langle\begin{array}{l}
x_{0} x_{1} \ldots . x_{m-1} \\
y_{0} y_{1} \ldots . . y_{m-1}
\end{array}\right. \\
& m_{1}=\frac{a_{0}}{b_{0}}\left\langle\begin{array}{l}
a_{0} a_{1} \ldots . . a_{m-1} \\
b_{0} b_{1} \ldots \ldots b_{m-2}
\end{array}\right. \\
& m_{2}=\frac{d_{0}}{e_{0}}\left\langle\begin{array}{l}
d_{0} d_{1} \ldots \ldots d_{m-2} \\
e_{0} e_{1} \ldots \ldots . e_{m-3}
\end{array}\right. \\
& : \\
& m_{k-2}=\frac{p_{0}}{q_{0}}\left\langle\begin{array}{l}
p_{0} p_{1} \\
q_{0} q_{1}
\end{array}\right. \\
& m_{k-1}=\frac{l_{0}}{m_{0}}\left\langle\begin{array}{l}
l_{0} \\
m_{0}
\end{array}\right.
\end{aligned}
$$

Where $\quad x_{0}, x_{1} \ldots x_{m-1} \quad$ and $y_{0}, y_{1} \ldots . y_{m-1}$ are a coefficients of a $m^{\text {th }}$-order characteristic equation $1+u(s) G(s)$ and left over coefficients are approximated as

$$
\begin{aligned}
& y_{j}=r_{j+1}-\alpha_{0} s_{j+1} j=0,1,2, \ldots . n-2 \\
& m_{j}=p_{j+1}-\alpha_{1} y_{j+1} j=0,1,2 \ldots . n-3 \\
& : \\
& : \\
& w_{0}=v_{0}-\alpha_{n-2} x_{1}
\end{aligned}
$$

For a setting of a range of constants of each controller, a given formulation of inequality needs to be considered.

$y_{0} ; r_{0} ; s_{0} ; \ldots m_{0} ; p_{0} ; w_{0} ; v_{0}>0$

\section{Model order reduction technique, Tuning procedure, Fuzzy controller strategy}

\subsection{Model order reduction technique}

The broad power system model can be approximated with an aid of the model order reduction (MOR) to the compact system, which maintains inherent properties of an original system. In so many decades, several order reducing methods were released [24-34] and in a course of a MOR technique a main poles of an earlier scheme are reserved while a lagging poles are rejected. Those methods can be used when the device has the certain number of roots to design the certain controller. The Fuzzy controller structure in Fig 3. The broad range of power (more order and maximum order) is determined by a limited device (low order or lower order) that makes its simple output unfortunate. A Model Order Reduction (MOR) method is employed. A key concept beyond the process of model orders reduction is to preserve a major pole of the entire model of a plant and to incorporate non-dominant pole with dominant poles in certain classes / slots according to the findings of control system designers. Let a single input transfer function (SISO) be linear 'n'systems

$k_{n}(s)=\frac{N(s)}{D(s)}=\frac{\sum_{i=0}^{m-1} b_{i} t^{i}}{\sum_{i=0}^{m} a_{i} t^{i}}$ 
Where $a_{i}$ and $b_{i}$ are called as scalar constants.

Let a reduced $k^{\text {th }}$ order model $(\mathrm{k}=1,2,3 \ldots \mathrm{m}-1)$ of an original high order system (14) is synthesized, as shown below :

$$
R_{k}(s)=\frac{N_{K}(S)}{D_{k}(s)}=\frac{\sum_{i=0}^{l-1} d_{i} p^{i}}{\sum_{i=0}^{l-1} e_{i} t^{i}}
$$

\subsection{Design of the power system model load frequency control system}

For the largest hydrothermal power systems including single area and 2 surfaces the IMC-PID controller can be used.

And load frequency controls are designed to adjust loads and variable parameters gradually and small. The droop characteristics are shown and the input and output is taken as shown in Fig. 4 for a single area reheat thermal power system. The mathematical model (see Fig. 4), includes a thermal and hydrothermal reheating device combination. Appendix A provides system parameters. Fig. 4shows a small model of hydro-thermal system operation of the agitation system.

$$
T(s)=\frac{G_{p 1}(s)}{\left(\left(G_{g 1}(s) G_{r 1}(s) G_{t 1}(s)\right) / R_{1}\right)+\left(\left(G_{T 1}(s) G_{T R}(s) G_{T W}(s)\right) / R_{2}\right)}
$$

Where $G_{g 1}, G_{r 1}, G_{t 1}$ are a steam regulator, tanks, turbines parallel to $G T_{11}, G T_{R 1}$ a hydro governor, tanks and power system turbines are $G_{T R 1}, G_{T W 1}$ is also the load-related turbine $G_{P 1}$.

\subsection{Tuning procedure}

The load frequency control approach pattern through a 2DOFIMC needs to adjust a desired output to two parameters ( $\alpha$ and ad). Normally, the big value results in slow moving closed-loop reaction and adequate strength, apart from uncertainties on a plant model and inadequate robustness on another hand the lesser value results in very fast-moving closed-loop reaction. A tuning of an LFC is just the adjustment between the closed loop reaction and stability. At last IMC-PID controller is directly related to both variables, so it is of practical significance to choose these two parameters.

IMC-PID decentralized architecture for hydro-thermal reheating systems in two areas

The decentralized LFC is more interesting in terms of its flexibility in a composition and execution due to an inborn nature of large power systems. It continuously provides fast adjustment between interconnected entities. A decentralized controller [6] was therefore designed for a hydrothermal two-area reheat system and no torque power is to be anticipated,

Therefore, a power of a tie line exchange is omitted only for a purpose of calculating a decentralized controller. Moreover, a local Bi bias coefficient should be multiplied for a plant model (equivalent to the single area heating and hydrothermal system) (26). Subsequently, an obtained high-order device feature must be reduced by a proposed Logarithmic technique and then a LFC can be designed in the unified Load Frequency Controller configuration for two reheat hydrothermal power systems. LFC can therefore be built separately for each region. Therefore, each area architecture must be taken into account, taking into account a linkage between areas. A hydrothermal power system is shown in a mathematical model by block diagram. Two areas of interconnection : area 1 consists of a hydrothermal and reheats thermal system, and area 2 also consists of. Appendix the shows a block diagrams and a system parameters. A distinction between LFC and two-area does not return a frequency of each area to its normal value, but a net switch over the tie-line should return to a reserved values. Thus, the composite calculation entitled area control error and a feedback variable is used. All symbols are a same as Fig. 5 except for a fact that a $\mathrm{P}$ tie is a deviation from a listed line power and a T12 is a coefficient of synchronization.

$A C E=\Delta P_{\text {tie }}+B_{1} \Delta f_{1}$
$A C E=-\Delta P_{\text {tue }}+B_{2} \Delta f_{2}$
$\Delta p_{\text {tie }}=\frac{T_{12}}{s}\left(\Delta f_{1}-\Delta f_{2}\right)$

In case of $\mathrm{B} 1$ or $\mathrm{B} 2$, area control errors of area 1 and of 2 are settings for a frequency biases, ACE1 and ACE 2. Also in a field I I 1,2) a feedback control is shown as

$u_{i}=-Q_{i}(s) A C E_{i}$

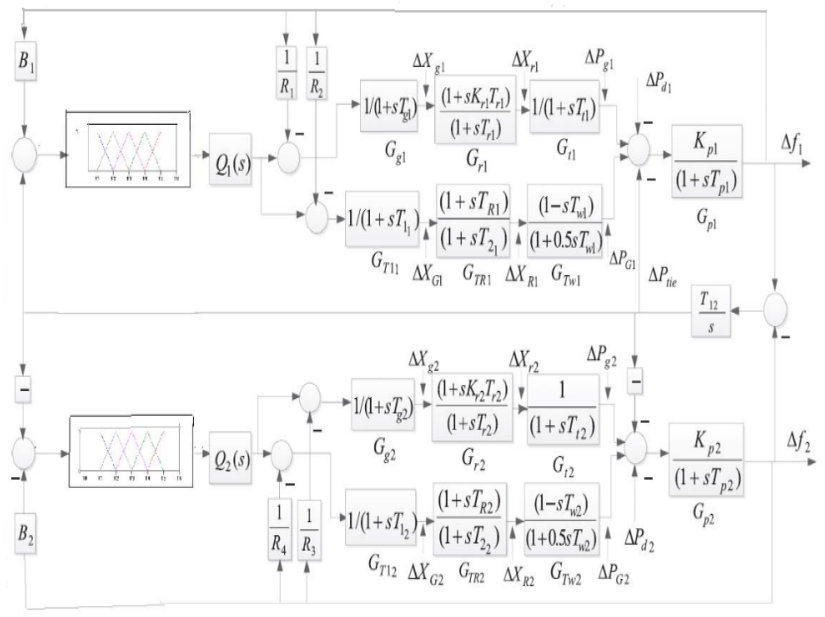

Fig.4 Block-diagram of two-area reheat hydrothermal power system 
Decentralized controller tuning can be assumed that an interchange power of a connection line is not available, $\Delta \mathrm{P}$ tie $=0$. A control signal generated is therefore as

$$
u_{i}=-Q_{i}(s) A_{i} \Delta f_{i}
$$

From a above review it is clear that a T(s) plant model, i.e. Eq. (27) by $\mathrm{Bi}$, is required to be multiplied and followed up on a same process as a one-area LFCPID tuning (refer Section 5.1). LFC can therefore be freely tuned for each region. Since interfaces are coupled, tuning parameters for each area should be taken into account. Thus, a mathematical model representation of field 1 can be written in two areas of a structure.

$$
p_{1}(s)=\frac{G_{p 1}(s) B_{1}}{\left(\left(G_{g 1}(s) G_{r 1}(s) G_{t 1}(s)\right) / R_{1}\right)+\left(\left(G_{T 1}(s) G_{T R 1}(s) G_{T W 1}(s)\right) / R_{2}\right.}
$$

\subsection{Fuzzy controller strategy}

In order to provide the internal functions to maximize (or) minimize and modifying Fuzzy control in an active models of wind turbine generators, a numeral values for membership functions must be calculated using the welldefined mechanism. Fuzzy droop signal input is a frequency deviation (f) and shift in a deviation of frequency ( $\mathrm{ft} /$ ) and an output control signal (PFLC) that changes an active injection setting. An inference method of type Mamdani is also used for fluid droops. A Membership features are called LN (BIG NEGATION), MN (MIDE NEGATION), ZON (ZZ), MP (MIDE POSTIVE) and LP (LARGE POSITIVE). In addition, symmetrical 7-segment member functions are triangular in order to obtain rapid answers from a control system. Table 1[40] provides for the rule base, consisting of 49 Fuzzy rules, to trace an input space to its output space. Rules of Fuzzy can be represented in an IF THEN format, and member feature parameters are set in a, b, and c. These parameters influence an output of fuzzy droops and a load control system includes $<$ b $<$ c $<$ Max. In addition to this, only one parameter that is called $d$ is needed for an output signal of a Fuzzy controller. All uncertain values have significant behaviour on a device frequency characteristic, e.g. a maximum frequency response slope and a setting time. In order to achieve desired frequency behaviour, it is therefore important to identify an optimum number of parameters. In other words, in this section, a problem is to find an optimum combination of uncertain values, namely a parameters for a membership function so that a micro grid reactions can improve. An output of a fuzzy controller does not rely on an input scale, according to a fuzzy logic controller theorem. This implies that it is not important if incoming signals are large or compact for a fuzzy logic controller. Indeed, fuzzy controller output is linked with a listed Fuzzy rules and membership parameters. The Simple Triangular membership ship functions for the FLC are shown in Fig 5 and 6.

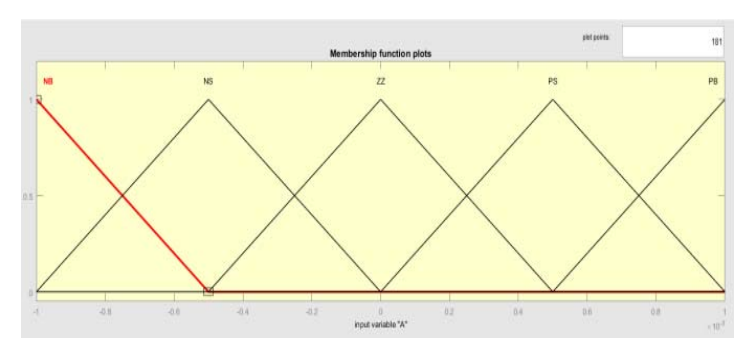

Fig.5 Input Membership Function of FLC

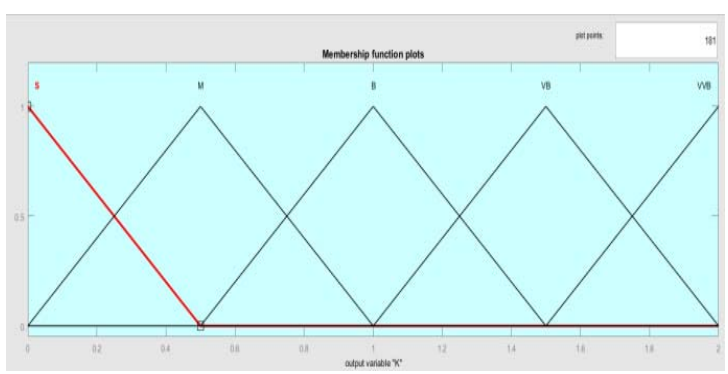

Fig .6 Output Membership Function of FLC

\section{Results and Discussions}

With the thought of Logarithmic approximations and $0.01 \mathrm{pu}$ load unsettling influence recreation is done with the qualities appeared in reference section for the LFC Model, thinking about all the regulators with the viability of the proposed Technique i.e IMC Fuzzy-PID Controller. Utilizing proposed technique, the recurrence deviations is immediately determined back to and has the better presentation in charge and damping of recurrence oscillations when contrasted and ordinary LFC and LFC with the proposed controller. Deviations in frequency for customary LFC models (with IMC-PID and proposed IMC-Fuzzy regulator) is appeared in Fig.7 to Fig10.

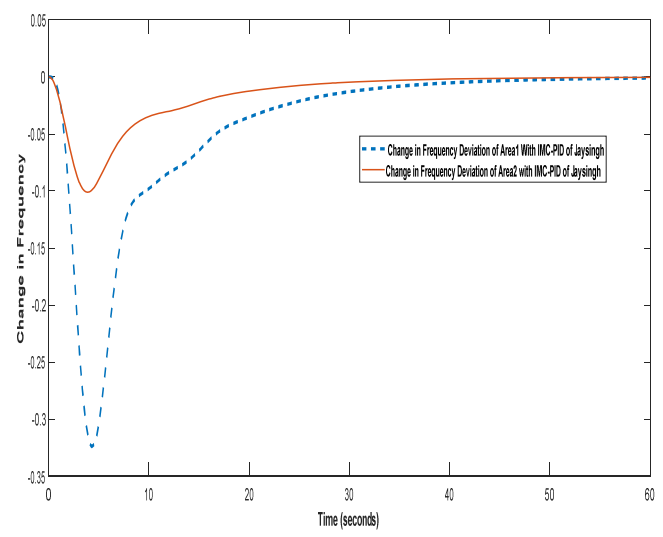

Fig .7 Change in Frequency deviation with IMC-PID Controller for Area1 and Area2 
These are existing results (Jaysingh) of frequency deviation of Area1 and Area2 in terms of Undershoot time of Area1 is -0.33 and Area 2 is -0.1 and their settling time is about $45 \mathrm{sec}$.

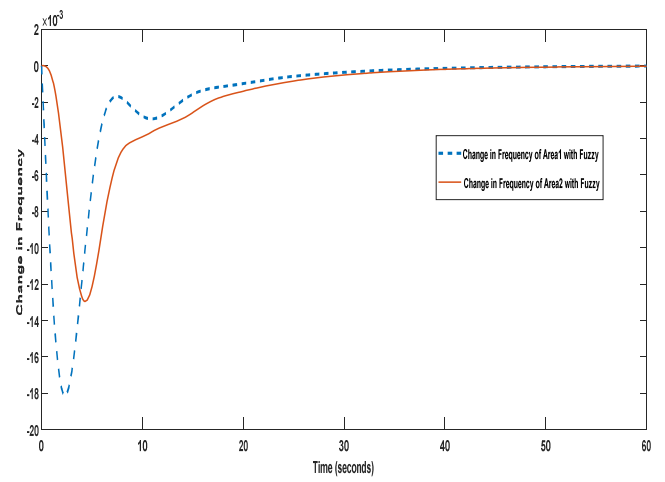

Fig .8 Change in Frequency deviation with IMC-Fuzzy Controller for area1 and area2

These are extension results with the proposed techniques of change in frequency deviation of Area1 and Area2 with fuzzy and their peak times are -0.018 and -0.012 , And their settling times are $30 \mathrm{sec}$.

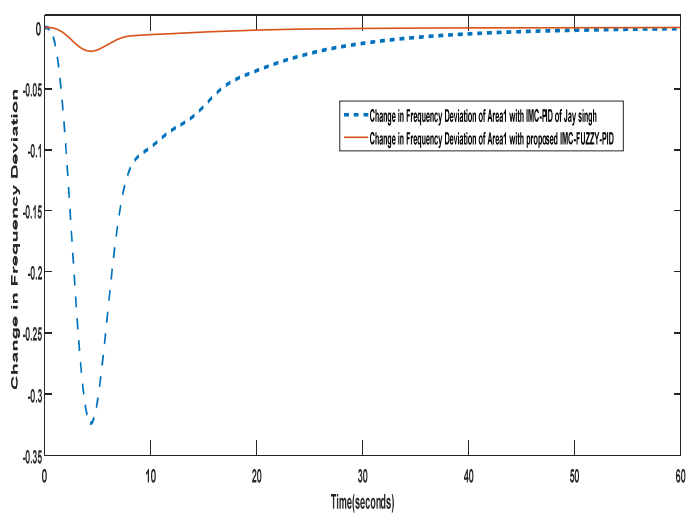

Fig .9 Comparative Studies with Jaysingh and the Proposed controller for Area1

It is the comparison of frequency deviation of Area1with existing (IMC-PID) and Proposed (IMC-FUZZY) and their peak times are -0.3 and -0.025 and settling times are $50 \mathrm{sec}$ and $30 \mathrm{sec}$.

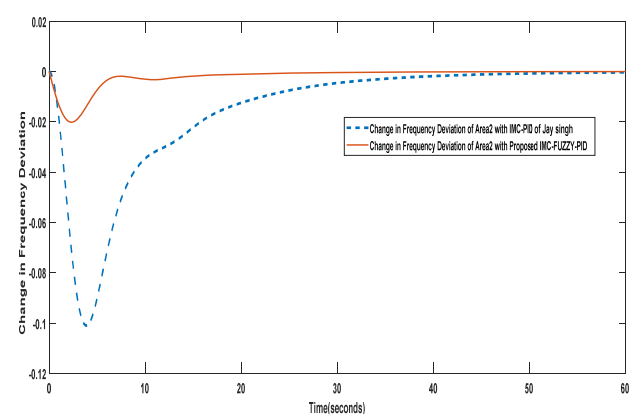

Fig .10 Comparative Studies with Jaysingh and the Proposed controller for Area2
It is the comparison of frequeny deviation of Area2 with existing (IMC-PID) and extension (FUZZY) and their peak times are -0.1 and -0.02 and their settling times are $50 \mathrm{sec}$ and $30 \mathrm{sec}$

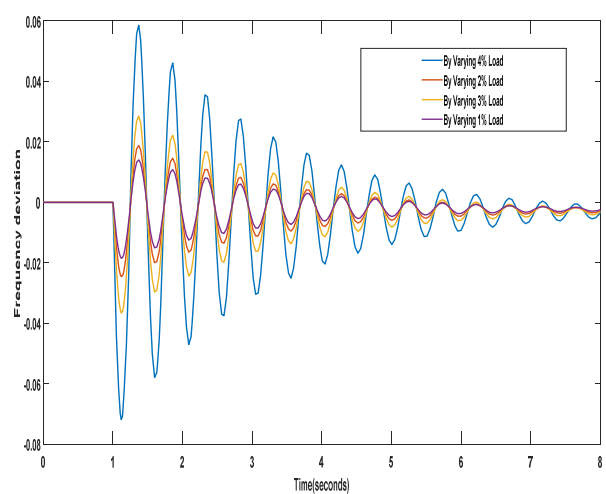

Fig.11 Change in frequency deviations of single area reheat hydro thermal system with varying loads.

These are change frequency deviations shown in Fig 11 for a single area reheat hydro thermal system with varying loads using the above IMC design considerations. These are having different peak times based on their variation of load and their settling time is 8 sec.

\section{Conclusion}

During unregulated experiments, an area control errors have been aggregated as a load fluctuations increase. FLC has a result that a variance in $\Delta \mathrm{f} 1, \Delta \mathrm{f} 2$ and $\Delta \mathrm{Ptie}$ are totally oscillatory if put in both zones for the phase load shift in area-1. In both areas of FLC with area-1, comparable deductions can be drawn in respect of equivalent phase load shifts. A reverse retorts are produced in both areas using FLC. Anomalies are small and oscillations are easily eradicated when FLC is put in either region. Compared with traditional and GA PI controllers, an implementation of a fuzzy controller provides an improved performance. A settlement time and rising time have dramatically decreased. A temporary is very easy to settle. A projected controller has been shown to be accurate and to increase device efficiency significantly.

\section{References}

1. W.Tan , IMC. IEEE Trans Power Sys, 25 (2010)

2. Guhadipayan, S. Banerjee, R.Provas kumar Krill,science direct, 5(2016)

3. W.Tan Tung, Eng Converse Manag science direct,51(2009)

4. S.Sahaj, H.Yogesh,. Elec Power Eng Sys, 81(2016) 
5. R.Asadur, S.Lalit chandra, S. Nidul, Institution of engineering and tech, 15(2015)

6. SC Tripathy, GS Hope, OP Malik,IEEE trans power sys,(1982)

7. S.Sahaj,H.Yogesh, science direct,28(2013).

8. R.Asadur , S.Lalit Chandra ,S.Nidul,IET Renew Power Gen, 8(2016)

9. Ch..Si-Lu, L. Xiaocong, T. Sing,T,80(2017).

10. T Liu, Ga F, 45 (2010)

11. P.Dash, S.Lalit Chandra, S.Nidul, Eectrical eng power sys ,68 (2015)

12. M.Hajiakbari Fini, R.YousefiGholam, A.Hassan Haes Institute of eng and tech, 5 (2016)

13. Sridevi, J., Amarnath, J., Govinda Rao, G. International Review on Modelling and Simulations ,4(2011)

14. L.Xiaocong, Ch. Si-Lu, T.Chek Sing, T.KokKiong. IEEE Elec eng power sys ,15 ( 2017)

15. S. Ganapathy, S Velusami. Elec eng tech ,4 (2009)

16. SK Nagar, SK Singh.Franklin institute, 341(2004)

17. Tan W. IEEE ,62(2011)

18. A Babu Suresh, Ch Saibabu, S Sivanagaraju.. Eng Sci Emerg.Technology,32 (2012)

19. CB Vishwakarma, R Prasad,Franklin institute 82(2014)

20. Jay Singh, Kalyan.Ch, CB Vishwakarma, IEEE 56 (2014)

21. MR Sathya, MMT Ansari, Elect Power Energy Sys, 46 (2015)

22. R.Asadur,S.Lalit Chandra ,S.Sinha,Institute of eng and tech, 39(2016)

23. Jay Singh, CB Vishwakarma,Ch.Kalyan,ISA trans.44(2017)

24. D.Puja,S.Lalit Chandra,S.Nidul.,Intitute journal elec eng power sys, 82(2016)

25. S.Swati, H. Yogesh,science direct ,85 (2014)

26. R.Shivakumar, R.Satheesh kumar,science direct 7(2016)

27. G Uma, MG Umamaheswari,Electr Power Syst Res, 56(2013)

28. P.Jayanta, AK Sinha tech ,IEEE 3(1990)

29. CB. Vishwakarma, Jay Singh, Ch.Kalyan IEEE, 32(2014)

30. Lakshmi Swarupa, M., Tulasi Ram Das, G., Sridevi, IEEE - ICREISG ,127 (2020)

31. Rajagiri, A.K., Mn, S.R., Nawaz, S.S., Suresh Kumar, T E3S Web of Conferences, 87.(2019)

32. Sridevi, J., Rani, V.U., Rao, B.L., IEEE conference ICECIE (2019)

33. Sridevi, J., Amarnath, J., Govinda Rao, G., International Review on Modelling and Simulations , (2011) 